\title{
Feature Matching in Iris Recognition System using MATLAB
}

\author{
Imran Naguru ${ }^{\# 1}$, Narendra Kumar Rao B ${ }^{\# 2}$ \\ \# Department of Computer Science \& Engineering, \\ Sree Vidyanikethan Engineering College (Autonomous), Tirupati, Chittor District-517102, A.p, India. \\ E-mail: ${ }^{1}$ imran567@gmail.com; ${ }^{2}$ narendrakumarrao@yahoo.com
}

\begin{abstract}
Iris recognition system is a secure human authentication in biometric technology. Iris recognition system consists of five stages. They are Feature matching, Feature encoding, Iris Normalization, Iris Segmentation and Image acquisition. In Image acquisition, the eye Image is captured from the CASIA database, the Image must have good quality with high resolution to process next steps. In Iris Segmentation, the Iris part is detected by using Hough transform technique and Canny Edge detection technique. Iris from an eye Image segmented. In normalization, the Iris region is converted from the circular region into a rectangular region by using polar transform technique. In feature encoding, the normalized Iris can be encoded in the form of binary bit format by using Gabor filter techniques. In feature matching, the encoded Iris template is compared with database eye Image of Iris template and generated the matching score by using Hamming distance technique and Euclidean distance technique. Based on the matching score, we get the result. This project is developed using Image processing toolbox of Matlab software.
\end{abstract}

Keywords - iris recognition; biometric identification; feature matching; iris normalization; image acquisition feature encoding; iris segmentation.

\section{INTRODUCTION}

Iris recognition system is a secure human authentication in biometric technology. This is one of the most accurate, reliable and leading technology for providing security. First Iris recognition system was proposed in 1939 by Dr. Frank and it has been implemented in 1990 by Dr. Daugman. At present Iris Recognition System has become more accurate and reliable biometric identification technology. Iris Recognition System consists of five stages to define or authenticate the user. They are Feature matching, Image acquisition. Feature encoding, Iris normalization and Iris segmentation. Image acquisition is the first stage in Iris recognition system. In this stage, first we capture two eye Images from CASIA database. The captured eye Images should be with good quality and high resolution for Image processing. Iris segmentation is the second stage in Iris recognition system. In this stage, Iris part is segmented from an eye Image by using canny edge detection technique and Hough transform technique. The third stage in Iris recognition system is Iris normalization. In this stage, the segmented Iris is converted from the circular region into a rectangular region by using polar transform technique and rubber sheet model. In Iris recognition system, the Feature encoding is fourth stage. In this stage, the normalized Iris is encoded in the form binary bit format and generates an Iris template by using Gabor filter technique.

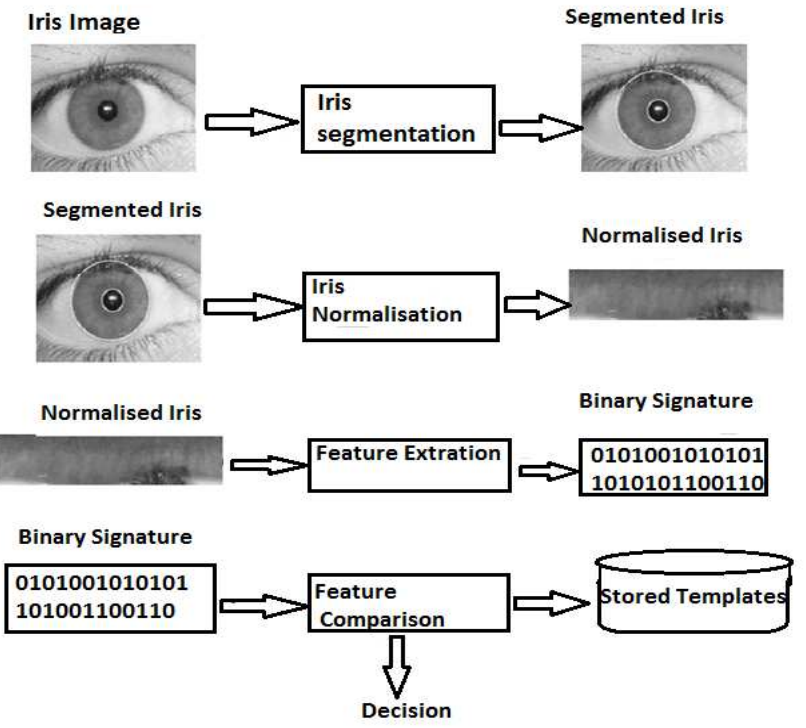

Fig. 1. Working flow of Iris Recognition System

Feature matching is final stage in Iris recognition. It is defined the integrity match score based on the two encoded Iris Images of Iris templates. Based on that matching score we get the result. 


\section{MAterial AND Method}

The Iris recognition system is defined in 5 stages. They are, Feature Matching, Iris Normalization, Iris Segmentation, Image Acquisition and Feature Encoding. The above Figure shows the Iris recognition system process.

\section{A. Methods for Image Acquisition}

In Image acquisition stage, we capture the Iris Images using different cameras or from the databases like CASIA and UBIRIS. The captured eye Image must have good quality along with a high resolution for Image processing. In table 1, we mentioned the different Image acquisition cameras with format and Resolution. Particularly Iris and pupil part must have good quality with high resolution for Image processing. Some Iris Images are available in the form of datasets which are freely available in the database. The Iris datasets are UBIRIS, CASIA, etc. This project implemented by using CASIA database.

TABLE I

CAMERAS USED FOR IMAGE ACQUISITION

\begin{tabular}{|c|c|c|}
\hline $\begin{array}{c}\text { Cameras used for Image } \\
\text { Acquisition }\end{array}$ & Format & Resolution \\
\hline Nikon E5700 camera & JPEG & $400 * 300$ \\
\hline Canon EOS 5D & JPEG & $800 * 600$ \\
\hline Iridian LG EOU2200 & TIFF & $640 * 480$ \\
\hline $\begin{array}{c}\text { AD-100 Iris Guard Dual } \\
\text { Eye autofocus camera }\end{array}$ & BMP & $1280 * 960$ \\
\hline $\begin{array}{c}\text { SONY DXC-950P 3CCD } \\
\text { Camera }\end{array}$ & PNG & $576 * 768$ \\
\hline LG Iris Access 2200 & BMP & $320 * 280$ \\
\hline $\begin{array}{c}\text { Panasonic BMET100US } \\
\text { Authentic cam }\end{array}$ & BMP & $320 * 280$ \\
\hline
\end{tabular}

\section{B. Methods for Iris Segmentation}

In segmentation stage, Iris part is segmented from an eye Image using different segmentation techniques. The Iris Segmentation techniques are Hough transform, Integrodifferential operator and gradient based edge detection, removal of eyelash \& eyelids and bisection methods. To segment the Iris, used Hough transform technique from an eye Image.

\section{Hough Transform Technique}

Caroline Houston [1] defined Iris segmentation is performed by using Canny edge detection and Circular Hough Transform techniques. Iris is segmented by using pupil and Iris center offset and Iris stretch methods. In Iris segmentation, Iris part is detected two circles from an eye Image, one is Iris boundary and other is sclera boundary. Hough transform is used to detect the centers and radius of two boundaries. In General Hough transform technique is used to detect geometric shapes in the form of circles, lines, parabola, and hyperbolas. For detecting circle of Iris, we used Circular Hough transform technique from an eye Image. Iris edges are detected by using a Hough transform technique. Iris segmentation technique performs well on most Iris Images for detecting Iris from an eye Image,
Sometimes system fails to detect Iris from an eye Image due to several eyelashes and deals with statistical interference and threshold. Eyelid can deal by implementing hyperbolic Hough Transform. In the result, suggested wavelet features and unique codes are generated for an individual eye Images. Iris segmentation can improve the Iris recognition system dramatically.

Samuel et. al. [2] defined horizontal and vertical derivatives in Iris recognition by developing Circular Hough transform technique. Provides a very accurate and reliable biometric technology to identify or authenticating the person. Iris consists of different Iris patterns for comparing one person with the other person. Iris templates are depends on reliability human Iris up to some level, which are identical twins. The eyelashes and eyelids are indicated by the lower and upper parts of Iris boundary, which are removed perfectly by using Hough transform technique. The accuracy of the system is calculated by converting Iris region into biometric template matching. The accuracy of proposed system is $95.6 \%$.

Sharma et al., [22] mainly focused on Hough transform method and developed efficient algorithm for Iris recognition in Iris segmentation stage. Over many years the Iris recognition provides a reliable verification and identification for an individuals and increased the biometric system accuracy. This process can captures an eye Image from user and segmented the Iris from an eye Image and normalized the segmented Iris using rubber sheet technique later we encode the normalized Iris by using Gabor filter technique and matching score is defined to the encoded Iris. The performance of this system depends on the segmentation and normalization. Firstly the Iris Images are selected from the database after removed the noises from an eye Image later the pupil and Iris boundaries are detected. The Iris region that is segmented for Iris normalization in order to minimize the dimensional inconsistencies. For Image processing and pattern recognition used Biometric recognition algorithms. Based on histogram threshold and gamma correction defined matching score to generate the result. By using Image processing toolbox of Matlab software this project is developed.

Amit et al., [23] applied Hough transform technique for detecting eyelashes, eyelids on Iris Image, but in order to improve the accuracy need to eliminate noise factors from the eye Image. Based on the physical characteristics and the human behaviour, the Biometric system is developed. Iris has a unique structure, which contains high accuracy and will be stable for each individual. A person recognized based unique features of Iris patterns. In the eye Image the Iris is found based on Iris patterns uniqueness. Iris recognition is defined in four stages; they are acquiring an eye Image from user, segmenting Iris, Feature extraction and Feature matching. To detect the eyelids and eyelashes, eyelashes detection algorithm is used. To identifying the lines and circles of particular radius from an eye Image used Hough transform technique. The performance of the Iris recognition is improved based on Iris Image of canny edge detection algorithm.

Verma et al., [24] uses Hough transform technique to segment the Iris by eliminating noisy and detecting Iris and pupil boundaries from an eye Image. Iris recognition system 
is the most accurate and reliable biometric identification system in the present situation. This system captures an Image of the human eye then the Iris Image is to be processed for segmentation, normalization and for the feature extraction process. For detecting the correct Iris need to remove the pupil noises reflection and the eyelashes present in Iris region correctly and accurately in Iris Segmentation stage. In Iris Normalization, the Segmented Iris is converted from circular region into rectangular region by using Rubber Sheet Model in order to minimize the dimensional inconsistencies in between the Iris regions. The normalized Iris region is converted into binary format and form a bit-wise biometric template produces the output like phase quantization by using the 1D Log-Gabor filters technique. By using Iris template we calculated matching score for authentication of an Iris Image.

Naveen et al., [25] presents the novel techniques in order to create the Iris recognition system for the analysis and used fusion mechanism. To detect the Iris boundaries used both Circular Hough Transform and Canny Edge detection techniques in the eye Image. In recent day's automatic identification provides different feature or the characteristic of the individuals in Iris recognition system which is more accurate and reliable for biometric identification. Wavelet transform technique forms a feature vector by determining Iris patterns. The two Irises are similar when quantized vectors is compared with the Hamming Distance and proved that system is quite effective.

\section{Integro-differential operator Technique}

R.B. Dubey et al. [3] proposed an approach for automatic segmentation that is based on different algorithms in Iris recognition system. It can localize the circular pupil region and Iris region by removing eyelashes and eyelids. The Iris recognition System is defined as most precise and reliable biometric authentic system. Patented algorithm is used for producing perfect Iris recognition rates in Iris recognition. These algorithms are based on linear search methods which are extremely slow to identify the process and raise the false acceptance rate. The Iris segmentation is achieved through by finding Iris boundaries as well as outer and inner boundaries of Iris region. The Iris region is approximately in between two circles, one is the pupil and other is sclera. In the eye except Iris remaining parts are normally removed from the lower and upper parts of the Iris region. Depending on the quality of eye Image, defined the success rate of Iris segmentation. Poor recognition rates will induce due to noisy or falsely data. This work is mainly focus on exact Iris Segmentation method for improving better biometric authentication system in widespread application areas. The proposed method achieves in 12.056 seconds of computation time in Iris segmentation but Hough transform method achieved in 248.23 seconds.

Maeva et al. [4] proposed an improvement method for Iris segmentation process. Firstly, the Iris boundaries are identified by using Integro differential operator method and identified eyelashes and eyelids are removed by using Linear Hough transform technique based on threshold. Iris segmentation method is improved in order to find and remove the eyelids and eyelashes with a precise method. Due to poor recognition of Iris regions the biometric template results will be corrupted. To avoid this drawback, Daugman's integrodifferential operator method is used.

\section{Eyelash Detection Technique:}

Yuan et al. [5] proposed a method for detecting eyelashes accurately. Eyelashes are two types; one is separable eyelash and the other is multiple eyelashes. The main intention is to find out the eyelash without affecting outside and inside boundaries of Iris Image. In this, eyelash detection is a very significant part in Iris segmentation process to get an accurate and desired results. Firstly, he is used local intensity minimum algorithm to detect the eyelashes, later it is detected based on the template of standard deviation and mean. Iris region consist of high-intensity values than Separable eyelash region. To obtain eyelash points, simple threshold can be used. Multiple eyelashes are overlapped in a small region. To detect multiple eyelashes, the standard deviation and means are used. In the result, it is proved that the eyelash noise detect ed with an proposed approach accurately.

Zemao et al. [6] proposed an eyelash recognition algorithm based on adaptive threshold. It is used for reducing eyelash influence on Iris recognition rate. Adaptive threshold is used to segment the eyelashes, according to gray distribution of the parabolic pixel point, upper eyelid parabolic moved down. In the results, we can observe that the improvement of the threshold is better in order to detect eyelash edges by comparing with traditional eyelash detection algorithm, it is not necessary to set up more parameters for the algorithm. It can embed with simplicity to maximize the result of eyelash segmentation. The algorithm reduces the three-dimensional searching space of the traditional algorithm with one dimension, So that the speed has been increased significantly. The eyelid detection algorithm is used for the eyelash threshold segmentation of the adaptive generation, and compared with the traditional algorithm which doesn't need to set up more parameters to segment effectively. The eyelash maximization can improve further aspects like the gray morphological operation. The palpebral eyelash interference will be better to remove seek for adaptive structure elements. The adaptive generation algorithm can improve the threshold to detect eyelash edges.

\section{Eyelid Detection Technique:}

Adam et al. [7] proposed two steps algorithm. One is possible edges are detected by selecting the inside area of the Iris. To refine parameters of a larger area, applied maximization at every parabola. The segmentation can be done manually. The performance evaluation of segmented Iris is compared with the manual segmentation using proposed method. More than $90 \%$ of the cases are satisfied with the results. In this, based on parabolic curve fitting proposed a new eyelid localization algorithm. Due to Iris texture, low contrast, the false detection is calculated by dealing with eyelashes. On a restricted Image area, we used the edge detection method. Parabolic arcs are estimated based on edges. A gradient maximization is applied on the parabola curvature of a larger Image area is restricted by using maximization of every candidate. Accurately is demonstrated by detecting the final boundaries.

Ismail et al. [8] proposed a simple algorithm for identifying boundaries of eyelids in an eye Image and 
efficient eyelid detection algorithm is applied on eye Image. Under less constrained Imaging conditions, the Iris Images are gained. Based on live-wire technique developed a detection algorithm for detecting eyelid. The eyelid detection algorithm main advantage is very simple computationally because the detection algorithms is compared with other prior eyelid algorithms. The Iris Image is determined in between outer Iris boundary and each eyelid by an two intersection points. The two intersection points are detected by using live-wire technique. In this, less constrained Iris Images accuracy is improved in Iris segmentation stage.

5. Non-Linear Circular Active Contour Technique:

Satish et al. [9] improved the efficiency and accuracy to the UBIRIS and CASIA V4.0 Iris databases with a proposed technique and also uses Geodesic Active Contour (GAC) technique for segmenting Iris from eye structures. Active colours can assume shapes and segment multiple objects simultaneously. In the survey most segmentation models assumed that the eyelid, limbic and pupillary boundaries are elliptical or circular in shape. The constant unique pattern of the Iris makes it a rich biometric characteristic for human recognition. The $1^{\text {st }}$ stage of Iris recognition is to keep a part of the actual Iris region in a digital eye Image. Iris segmentation stage is significant to achieve Iris pattern which are falsely represented in an Iris recognition will corrupt the biometric templates. In the result, less recognition rates of data is generated.

Mohammed et al. [10] proposed a scheme for a non-ideal Iris Images to find the exact pupil boundary. The Iris recognition system affected significantly in non-ideal Iris Images, and also affected the segmentation accuracy. To segment the pupil shape proposes an accurate and efficient method by using active contour and morphological operation methods. The rough pupil boundary is detected by using Morphological operations method. To identify the actual boundary, Active contour method is applied. The inner Iris boundary is segmented by proposed method in real shape, which is more accurate and more reliable to contribute a rich Iris recognition. The main advantage of the active contour is to achieve more accuracy and efficiency by generating the more accurate active contour of pupil boundary than the circular and elliptic approximation. In the result, high-level accuracy is found on CASIA V.4 database.

\section{Methods for Iris Normalization}

In Iris recognition system, the Iris normalization is third step. In this, the localized Iris is converted from the circular region into a rectangular region. It has some techniques to normalize the segmented Iris. The normalization techniques are the non-linear model, polar transform and rubber sheet model. In this, we used Rubber Sheet technique and polar transform technique to normalize the Iris.

\section{Non-Linear Technique}

Chul et al. [11] proposed non-linear tensile properties of Iris patterns. It can detect outer and inner regions of Iris by eliminating specular reflections, eyelash regions and eyelid. To Normalize the Segmented Iris, normalization technique is applied. Which converts the Iris from the circular region into a rectangular region. In Iris region, we can able to allocate the tracks Non-Linearly and dynamically, in which each track can be extracted by using Gabor filter technique and forms a binary bit code ( 0 and 1$)$. The extracted Iris codes are used for enrolment to identify the person. The proposed method can be defined in two steps. In step 1, Iris patterns are automatically detected with Non-Linear positions by changing illumination conditions in a radial direction when contracted or dilated the pupil. In step 2, differentiated tensile properties of Iris patterns individually and this method determines based on the user dependent method of Non-Linear dynamic track allocation, in which the Iris recognition accuracy is greatly improved. Based on the linear rubber sheet model the proposed method will have high accuracy and efficiency than conventional methods which will use consistent track allocation.

Zhenan et al. [12] defined a novel method for correcting Non- Linear Iris Deformation. In this, Iris Images are corrected by an addictive deviation between Non-Linear and Linear Iris stretch. The corresponding distance under linear stretch plus an addictive deviation is calculated in between an Iris region to pupil boundary. In the result, the effectiveness algorithm is demonstrated based on two databases with non-linear deformation. In the survey, lower Equal Error Rate (EER) is defined by using proposed Iris deformation algorithm by comparing with two Non-Linear and linear normalization methods. It can make the system more robust in a real environment. The proposed method is more efficient than other approaches.

\section{Polar Transform Technique:}

Anicham et al. [13] used different techniques for Iris recognition system. It is consists of five stages. They are Feature matching, Iris normalization, Iris segmentation, Image acquisition and Feature encoding. Each step is defined by using various techniques of Iris recognition system. To segment the pupil and Iris boundaries proposed a method that are detected all boundaries of Iris by using Hough Transform technique from an eye Image. Daugman's rubber sheet model defined the Iris normalization from segmented Iris. The active contour transform technique is extracted or encoded the normalized Iris. To classify the Iris Image SOM, LDA techniques are used. Iris recognition is a secure and sufficient than using the user id and security code and the interlopers prevented by the malicious action. This experiment can be executed by using MATLAB.

Mayank et al. [14] proposed an algorithm to increase the speed and the accuracy of Iris recognition, indexing, match score fusion and quality enhancement, for Iris segmentation. In this, the modified Mumford-Shah functional is used to propose Curve evolution approach to segment the Iris. Multiple enhanced versions of the Iris Images are produced in Iris Segmentation by using Different improvement algorithms which are concurrently applied. SVMBLA enhanced the local region from global region. The highquality Iris Image is created by using SVMBLA. The highquality Iris Images are encoded from two distinct features. Euler number and 1-D log-polar Gabor filter encode the local topological feature and global textural features. An intelligent fusion algorithm is improve the performance of matching score and textual score in Iris recognition system and reduces the FRR. Supports accurate and efficient Iris identification in an indexing algorithm. Performance of 
Identification is verified and validated by using a proposed algorithm and other algorithms are comparing with proposed algorithms by using the UBIRIS, ICE 2005, and CASIA Version 3 Iris database.

\section{Rubber Sheet Model technique:}

Qureshi et al. [15] proposed conventional Iris recognition method with three serious modifications which are very efficient and secure for Iris recognition. To Segment the pupil from eye Image by using canny edge detection technique and which is specially designed for handling pupil occlusion problems. Rubber sheet model is used for feature extraction, which can encode the normalized Iris into binary bit format. Feature matching determines the matching score. In this, some serious modifications are done to the rubber sheet model and k-nearest method for achieving accuracy and efficiency in Iris recognition system. The average Iris recognition time is found as $0.8254 \mathrm{sec}$, which is very less when compared to state of other techniques.

Rana et al. [16] proposed Daugman's rubber sheet model based on Independent Component Analysis (ICA) and Principle Component Analysis (PCA). Iris recognition is a fast biometric technology to authenticate or identify the person based on unique pattern of Iris recognition. The proposed algorithm increases the accuracy and reliability by comparing over two hundred million of Iris Images. A working prototype of techniques is used to implement the Iris recognition. In this work used three different algorithms in order to increase the efficiency of Iris recognition. The algorithms are Daugman's rubber sheet model, PCA and ICA. The UBIRIS database is used to capture the Iris Images, in which each database there are 230 sets of Images by consists of 5 Images in each set of a single person. In the result, efficient results are obtained by comparing with existing methods.

\section{Methods for Feature Encoding:}

In Iris recognition, the Feature Encoding is fourth stage. In this stage, the encoded binary bits are formed by normalizing the Iris. It has some techniques for encoding the normalized Iris. The techniques are Gabor filter, Cumsum based CPA and Ridge let. In this, we used Gabor filter technique for encoding normalized Iris into binary bit format.

\section{Gabor Filter Technique}

Avila et al. [17] proposed Hamming distance and Gabor filter techniques for feature matching and feature encoding. To develop biometric technique used Hamming distance and Gabor filters technique to describe different approaches. The wavelet transform is applied for zero-crossing representation and Iris signatures are defined in two ways: one is based on annular region and another is based on a circle of the Iris and also compared with some other metrics in the results. Proposed approach is a scale invariant, a translation and rotations are also worked in 0 crossing representation of wavelet transform. In the result, the Classification up to $99.6 \%$ of Success Rate is achieved and down to $0.12 \%$ of Equal Error Rate achieved and contained low false rejection rates, null false acceptance rates.

Dhinesh et al. [18] defined the Feature Matching, Feature Extraction and Iris segmentation. In Iris segmentation, to detect the outer and inner boundaries proposed a method for Iris curves. In feature extraction, proposed a curve let transform method for detecting the feature points from segmented Iris by using log-Gabor filter technique to produce a rotation invariant descriptor at every detection point. In feature matching, proposed a technique based on PFM method and compared with 2 sets of feature points by using local feature point and position of every point. In the result, achieved more accuracy percentage in curve let transform than log-Gabor filter curve let transform.

\section{E. Methods for Feature Matching:}

In Iris recognition system, The Feature matching is last stage. In this, feature matching is defined by the integrity match score based on the two encoded Iris Images. It has some techniques to define the match score. The feature matching techniques are hamming distance, Euclidean distance and K-NN classifier.

\section{Euclidean Distance Technique}

Win et al. [19] used different methods for Iris recognition system and defined Feature matching, Iris normalization, Iris segmentation, Image acquisition and Feature encoding stages for Iris recognition system by using different techniques. In Image acquisition, Eye Images are captured from CASIA database and eyelashes, eyelids are removed from an eye Image by using Hough transform method in Iris recognition. In Iris normalization, the Iris part is normalized by using rubber sheet model to minimize the dimensional inconsistencies between Iris regions. In feature extraction, one- dimensional object detection method is used. In feature matching, Euclidean distance method is used and compared with two Iris textures. In the result achieved more accuracy in Iris segmentation and feature extraction by reducing the computational time. The performance of Iris segmentation and feature extraction is defined based on accuracy of Iris recognition.

Sahoo et al. [20] proposed a feature matching technique for Normalized Iris based on wavelet decomposition of optimized Iris. The unique Iris patterns of the people, which are highly stable from starting birth to death duo to this defined Iris recognition system. For this we proposed many methods and techniques from last few years. In this, the evaluation of normalized correlation is an optimum sub band decomposition, the accuracy is improved by determining the Iris recognition system and made the robustness and simpler by increasing the Iris recognition system. The matching score enhances the selective matching Iris set and reduces FRR of registered user and FAR of the imposter in the recognition system. In the result, reliability and accuracy of Iris recognition system are improved efficiently.

\section{Hamming Distance Technique}

Hesham et al. [21] proposed an algorithm to generate a Iris template for Iris recognition system by using most consistent feature data. A new metric measure formula is used to calculate hamming distance in Feature matching and which the noisy level presented in the considered images. In this, to increase the accuracy of an Iris recognition system required many eye Images of same person and to generate final Iris template used an algorithm in which base templates are mixed with the given Iris templates set. In this work, revealed a reduction in size by using 45 persons of 450 Images from MMUI database almost $78 \%$ and increases in 
verification speed around $80 \%$ while maintaining $99.7 \%$ accuracy of matching.

Gayathri et al. [26] used hamming distance measurement to authenticate the users by comparing quantized vectors. In this, discussed Feature matching, Iris normalization, Iris segmentation, Image acquisition and Feature encoding are based on user Iris. Circular Hough transform methods are used to identify the Iris boundaries in eye Image. Image registration technique is used to normalize the segmented Iris. For Iris Image registration, correlation base method is used. Registration method adjusts points with corresponding points by replacing one object view with another object view to determine the geometrical transformation. In feature encoding phase, the normalized Iris is encoded by using 2-D Gabor filter technique. In this paper developed a new Iris recognition system by using phase correlation technique to compare the two eye-Images. These techniques has been calculated individually for several different applications. In the result, the proposed algorithm increases the security by using encryption techniques and encourages the performance of Iris recognition system.

Prashanth et al. [27] described an efficient biometric security based on Iris recognition system. Iris Images are verified or identified by using Integral Wavelet Transform (IWT) technique. Iris recognition is one of the most secure and authentic method for human recognition by comparing with other biometrics. Integro-Differential operator method is used to identify the Iris and pupil boundaries in an eye Image. Extracted the Normalized Iris by using Discrete Wavelet Transform (DWT) and IWT. For Feature matching of two Iris feature vectors used Hamming Distance technique. In the result, we detected that the values of computation time, ERR, FRR and FAR are required to improve Human Iris Recognition system as compared with the IWT to DWT. The Iris recognition system is implemented by using DWT and IWT. The efficiency and computation speed is improved based on Iris recognition system. The mean of Hamming Distance is calculated for making decisions and selected only one matched Image from out of seven sets of Iris Images. The efficiency algorithm is improved the Iris recognition system.

\section{RESUlTS AND DisCUSSION}

The Iris recognition system is defined in five steps. They are Feature matching, Iris normalization, Iris segmentation, Image acquisition and Feature encoding. In literature survey we defined all techniques of Iris recognition system based each stage. In this, Hough transform technique is used for Iris Segmentation, for normalizing the Iris used rubber sheet model, for feature encoding used Gabor filter technique and for feature matching used Hamming distance technique.

\section{A. Block Diagram}

Iris recognition system consists of five stages. They are Feature matching, Iris normalization, Iris segmentation, Image acquisition and Feature encoding. In Image acquisition, the eye Image is captured from databases of eye Images, the Image must have good quality with high resolution to process for next steps. In Iris segmentation, detected the Iris region from an eye picture. The Iris part is detected by using Hough transform technique. The segmented Iris is converted from the circular region into a rectangular region by using polar transform technique in Iris normalization stage. In feature encoding, the normalized Iris can be encoded in the form of binary bits by using Gabor filter techniques. In feature matching, the encoded binary bit form of Iris template is compared with database eye Image of Iris template, based on the matching score of both Iris templates output is displayed.

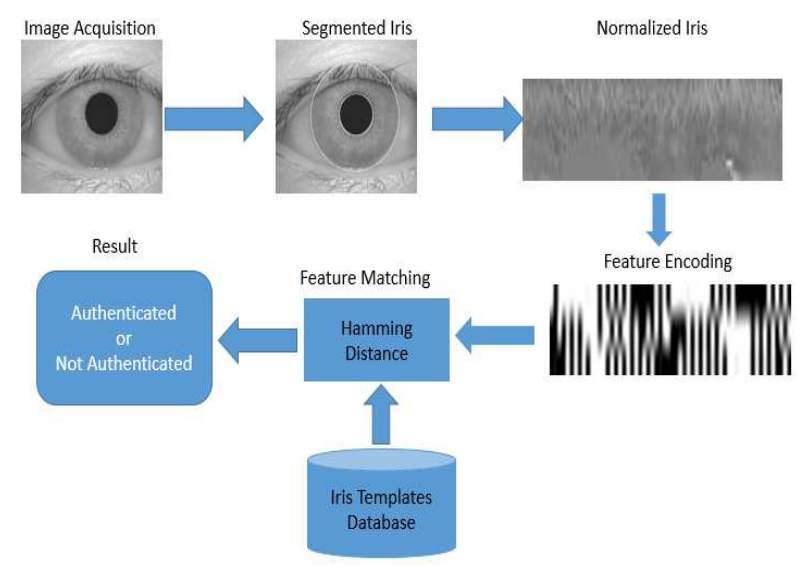

Fig.2. Working process of Iris recognition system

Steps: Iris Recognition system

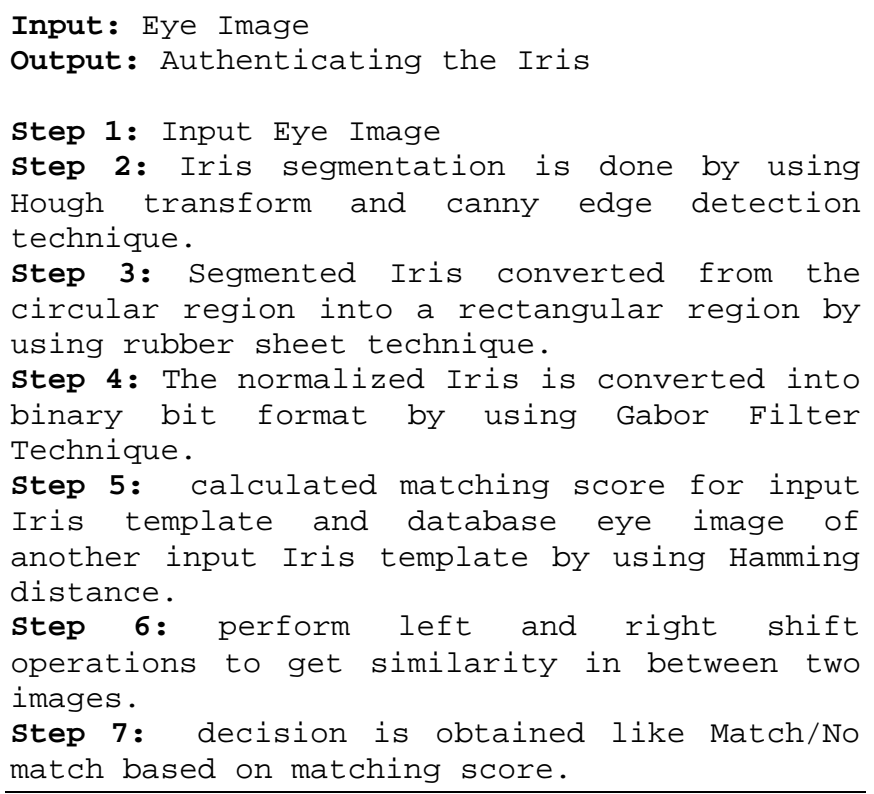

\section{B. Case Study:}

The Iris recognition system is defined in five stages. They are Feature matching, Iris normalization, Iris segmentation, Image acquisition and Feature encoding. For example, Fig. 1 shows Iris recognition system process. These all stages are explained clearly below.

\section{Image Acquisition}

In Iris Recognition System, Image acquisition is first stage. In this stage, we capture the Iris Images using different cameras or from database. The capture eye Image must have good quality with high resolution for Image 
processing. The different Image acquisition cameras are mentioned in table 1 with format and resolution. Particularly Iris and pupil part must have good quality with high resolution for Image processing. Some Iris datasets are available in database to test Iris recognition. The available Iris datasets are UBIRIS, CASIA, etc.

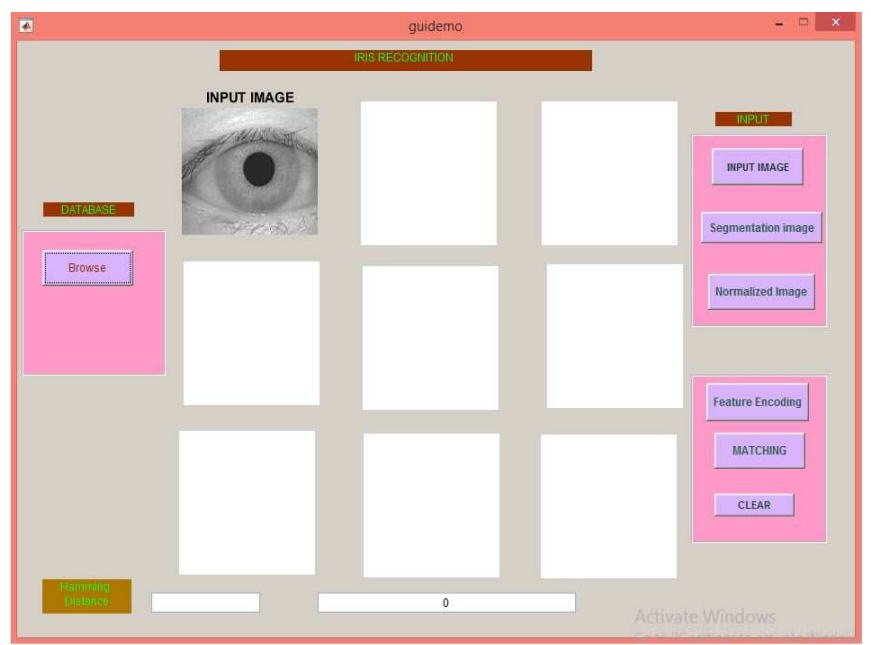

Fig.3. Output Image Acquisition

\section{Iris Segmentation:}

In Iris recognition system, the Iris segmentation is second stage. In this stage, the Iris is segmented from an eye Image by using different segmentation techniques. The segmentation techniques are Hough transform, Integrodifferential operator and gradient based edge detection, removal of eyelash \& eyelids and bisection methods. The Iris part is detected by eliminating the upper \& lower boundaries of eyelids \& eyelashes and central part of pupil boundary. In this we used Hough Transform Technique.

\section{Algorithm 1: Detecting the boundary of Pupil}

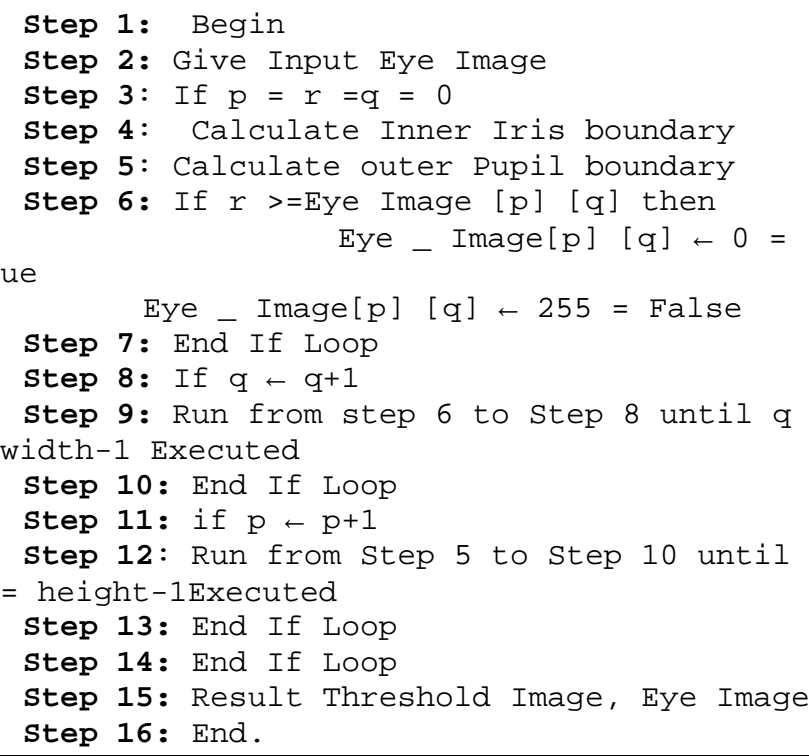

\section{Hough Transform technique:}

Hough transform technique is defined the intensity values of an eye Image by calculating the first derivatives of edge map and detected the outer Iris boundary and pupil boundary from the Hough space and edge map. From each edge point the parameters of the circles are passed. The radius $r$, centre coordinates $\mathrm{x}_{\mathrm{c}}$ and $\mathrm{y}_{\mathrm{c}}$, which are defined and representing parametric circle equation. It is given below:

$$
\left(X-X_{C}\right)^{2}-\left(Y-Y_{C}\right)^{2}=r^{2}
$$

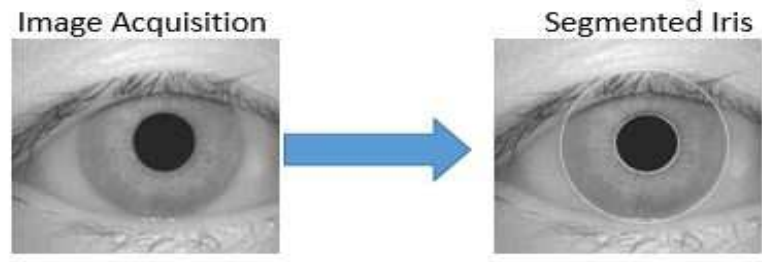

Fig.4. Segmented Iris

The equation of parametric circle representation:

$$
\begin{aligned}
& x=x_{c}+r \cos \theta \\
& y=y_{c}+r \sin \theta
\end{aligned}
$$

The Line equation is:

Where,

$$
r=x \cos \theta+y \sin \theta
$$

$r$ is the distance between origin and line $\theta$ is the angle of the vector

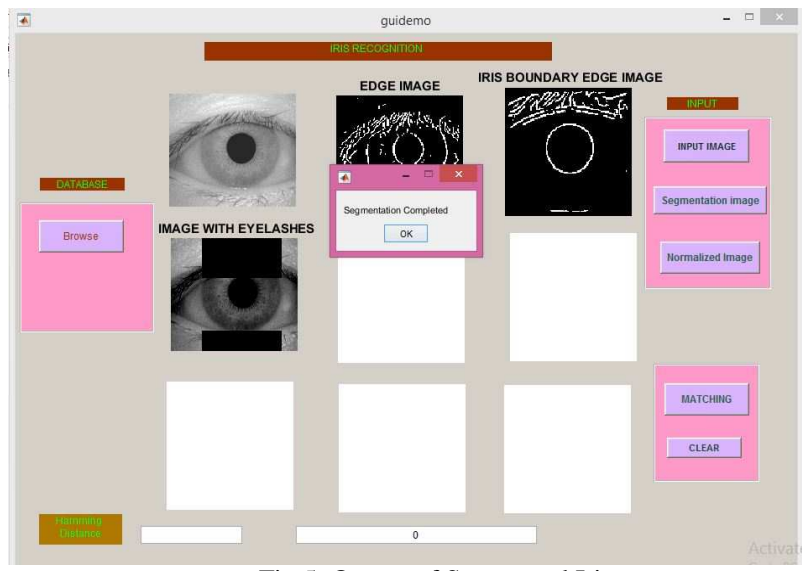

Fig.5. Output of Segmented Iris

Algorithm 2: Detecting Iris boundaries

Step 1: Begin

Step 2: Take Input - Image (Input Eye [] [], Iris _ r, pupil _ r, width, Height, $p_{C}$, $q_{c}$, )

Step 3: Consider $r 1=0$, pupil $-r=$ pupil

- r, Iris _ r= Iris_r1.

Step 4: Initially take $\mathrm{p}=0$

Step 5: do $\mathrm{p} \leftarrow 0$

Step 6: Initially take $q=0$

Step 7: do $q \leftarrow 0$

Step 8: Calculate radius using $\left(p-p_{c}\right)^{2}+(q-$ $\left.q_{c}\right)^{2}=r 1^{2}$

Step 9: If $r 1<p u p i l \_r 1$ or $r 1>\operatorname{Iris} r 1$

$$
\text { True: Eye }[p][q]=0
$$

Step 10: end if Loop

Step 11: Display Input _ Eye

Step 12: End 


\section{Iris Normalization:}

In Iris recognition system, Iris normalization is third stage. In this stage, the segmented Iris is converted from circular region into rectangular region. It has different techniques to normalize the segmented Iris. The normalization techniques are non-linear model, polar transform and rubber sheet model. In this we used Rubber Sheet Technique.

\section{Rubber Sheet Technique:}

Once Segmented the Iris, the next stage is Iris Normalization. In this the Segmented Iris is converted from circular region to rectangular region by using rubber sheet technique.

Iris Normalization is a process of converting the Iris into its polar equivalent. The centre of the pupil is identified and removed from an eye Image, and radial vectors are passing through the Iris region. The number of points are chosen in each radial line with the radial resolution. The Iris region is defined as the number of radial lines and angular resolution going around Iris region.

$$
r^{\prime}=\sqrt{\alpha \beta} \pm \sqrt{\alpha \beta^{2}-\alpha-r_{I}^{2}}
$$

With

$$
\begin{gathered}
\alpha=o_{x}^{2}+o_{y}^{2} \\
\beta=\cos \left(\pi-\arctan \left(\frac{o_{y}}{o_{x}}\right)-\theta\right)
\end{gathered}
$$

Where $r$ is the radius of Iris

$\mathrm{O}_{\mathrm{x}}, \mathrm{O}_{\mathrm{y}}$ is Displacements center of pupil to Iris

$\theta$ is the angle

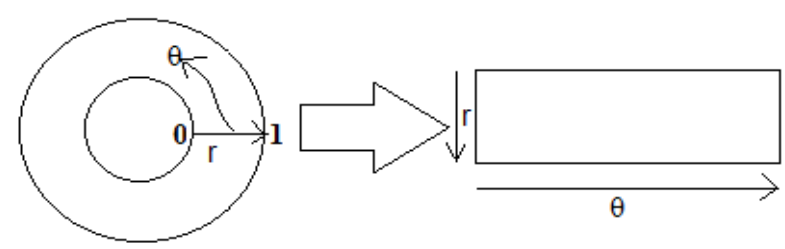

Fig.6. Unwrapping the Iris

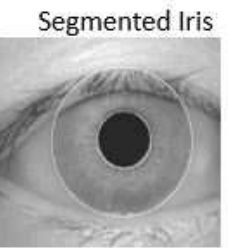

Normalized Iris

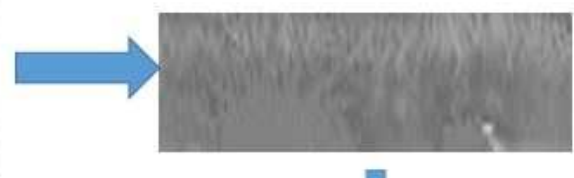

Fig.7. Normalized Iris

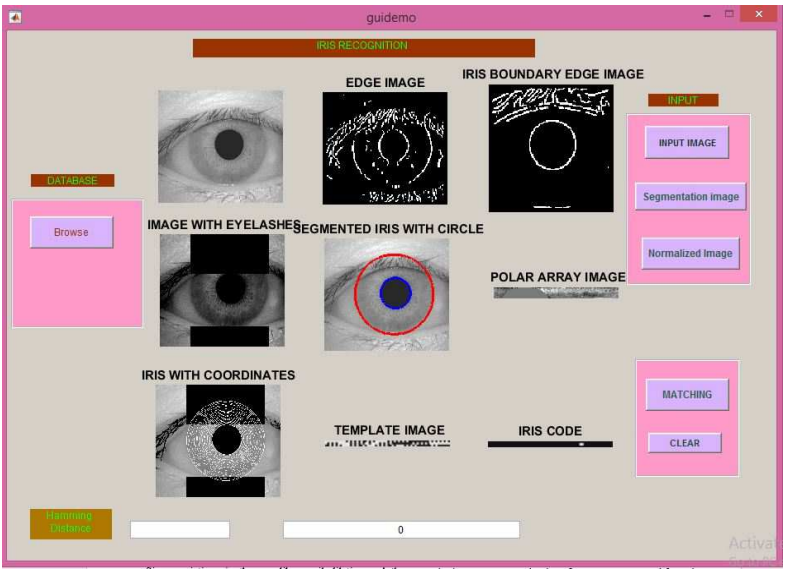

Fig.8. Iris Normalization and Feature Encoding

\section{Feature Encoding}

In Iris recognition system, the feature encoding is fourth stage. In this stage, the normalized Iris is encoded in the form of binary bits $(0 \& 1)$. It has different techniques for encoding the normalized Iris. The techniques are Gabor filter, Cumsum based CPA and Ridge let. In this we used Gabor Filter Technique.

\section{Gabor Filter Technique:}

The most discriminating features of Iris is created an Iris template. Extracted the feature extraction is done by Gabor filter technique to encode the normalized Iris region into binary bit format. A Gabor filter technique is developed by Gaussian and it is a sine wave modulated. Extracted the unique features of Iris once applied on entire Image. Feature encoding is processes of converting the normalized Iris into binary bit format by using 1D Gabor wavelets transform technique. In this, Iris normalisation is done by using polar coordinates, the polar form filter equation is given below

$$
H(r, \theta)=e^{-i w\left(\theta-\theta_{0}\right)} e^{-\left(r-r_{0}\right)^{2} / \alpha^{2}} e^{-i\left(\theta-\theta_{0}\right)^{2} / \beta^{2}}
$$

Where $(r, \theta)$ specify that center frequency of the filter $(\alpha, \beta)$ specify effective width and lengh

The Gabor filters technique defines output in order to compress the data into four levels. Each possible quadrant data in the complex plane is quantised by the phase information.

$h_{\{\mathrm{Re}, \mathrm{Im}\}}=\operatorname{sgn}_{\{\mathrm{Re}, \mathrm{Im}\}} \int_{\rho} \int_{\phi} I(\rho, \phi) e^{-i w\left(\theta_{0}-\phi\right)} e^{-\left(r_{0}-\rho\right)^{2} / \alpha^{2}} e^{-\left(\theta_{0}-\phi\right)^{2} / \beta^{2}} \rho d \rho d \phi$

where, $h^{\{R e, I m\}} \mathrm{Im}$ is the imaginary part and Re is the real part, depending on quadrant of Iris assign the values 1 or 0 , in dimensionless polar coordinate system $\mathrm{I}(\rho, \theta)$ is lies in raw Iris image.

Using imaginary and real values, extracted the phase information by encoding the bit pattern.

$$
P(r, \theta)=\tan ^{-1}\left(\frac{\operatorname{Im} \operatorname{Ig}(r, \theta)}{\operatorname{Re} \operatorname{Ig}(r, \theta)}\right)
$$




$$
I_{P}[r, \theta]=\left\{\begin{array}{c}
{[1,1] \text { if } 0^{\circ}<P(r, \theta) \leq 90^{\circ}} \\
{[0,1] \text { if } 90^{\circ}<P(r, \theta) \leq 180^{\circ}} \\
{[0,0] \text { iff } 180^{\circ}<P(r, \theta) \leq 270^{\circ}} \\
{[1,0] \text { iff } 270^{\circ}<P(r, \theta) \leq 360^{\circ}}
\end{array}\right\}
$$

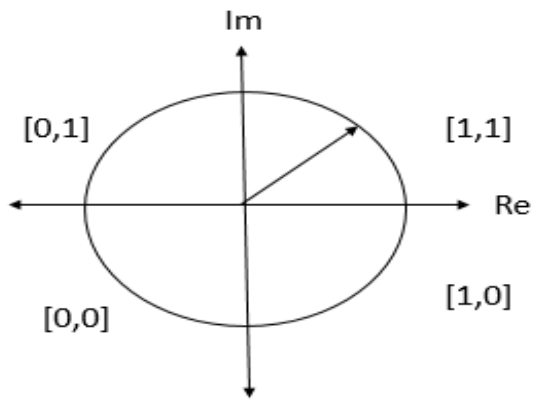

Fig.9. Phase Quantisation

The number of filters in the radial resolution is lesser than angular resolution. Total number of bits in the Iris template will be $\mathrm{n}$ times more than Iris template. Parameters and centre frequencies of modulating Gaussian functions must be detected the number of filters.

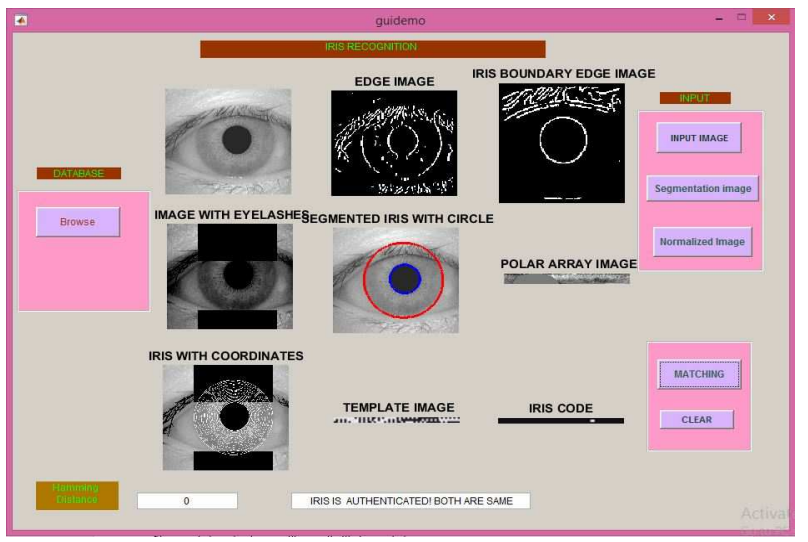

Fig.10. Feature encoding

\section{Feature Matching:}

This is last step in Iris recognition system. In this step, feature matching is defines the integrity match score based on the two encoded Iris images. It has some techniques to define the match score. The feature encoding techniques are hamming distance, Euclidean distance and K-NN classifier. In this we used Hamming Distance technique.

\section{Hamming Distance technique:}

Based on two Iris templates calculated matching score by using the Hamming Distance which gave a measure of how many bits disagreed.

Hamming distance is calculated by using two Iris templates, Iris template bits are shifted one shift right and one shift left to determine rotational inconsistencies. Two Iris images of the same eye will be determined by rotational inconsistencies with the difference of maximum angle between same Iris images.

Bit shift concept is applied to get approximate matching score by shifting one bit left and one bit right.

$$
\begin{aligned}
& \text { Template1 } 100011001001 \\
& \text { Template } 2001100100110 \Rightarrow H D=0.83 \\
& \leftarrow \text { Shift2bitsleft } \\
& \begin{array}{l}
\text { Template } 1001100100110 \\
\text { Template } 2001100100110
\end{array} \Rightarrow H D=0.00 \\
& \rightarrow \text { Shift 2bitsright } \\
& \begin{array}{l}
\text { Template1 } 011000110010 \\
\text { Template } 2001100100110
\end{array} \Rightarrow H D=0.33
\end{aligned}
$$

\section{Results}

Iris recognition system is developed by using Image processing toolbox of MATLAB software. In this project we used canny edge and Hough transform algorithms to find the Iris region. Daugman's algorithm applied to convert the segmented Iris from circular region into rectangular region. The normalized Iris is encoded in the form of binary bits $(0$ $\&$ 1) by using Gabor filter technique. In feature matching defines the integrity match score based on the two encoded Iris Images by using Hamming distance technique. In this we compared two (CASIA and UBIRIS) dataset of eye images by calculating the FAR and FRR. The formulas of FAR and FRR given below

$$
\begin{aligned}
& F A R=\frac{\text { WronglyAcceptedIndividuals }}{\text { TotalNuumberofWrong Matching }} \\
& F R R=\frac{\text { WronglyRejectedIndividuals }}{\text { TotalNuumberofCorrectMatching }}
\end{aligned}
$$

TABLE II

FAR AND FRR VALUES BY APPLYING HAMMING DISTANCE TECHNIQUE

\begin{tabular}{|c|c|c|}
\hline Threshold & FAR\% & FRR\% \\
\hline 0.12 & 0.000 & 98.947 \\
\hline 0.18 & 0.000 & 81.977 \\
\hline 0.24 & 0.000 & 36.980 \\
\hline 0.30 & 0.000 & 9.548 \\
\hline 0.36 & 0.005 & 0.827 \\
\hline 0.42 & 5.859 & 0.000 \\
\hline 0.48 & 90.549 & 0.000 \\
\hline 0.54 & 99.755 & 0.000 \\
\hline
\end{tabular}

TABLE II

FAR AND FRR VALUES BY APPLYING EUCLIDEAN DISTANCE TECHNIQUE

\begin{tabular}{|c|c|c|}
\hline Threshold & FAR\% & FRR\% \\
\hline 0.18 & 0.000 & 92.407 \\
\hline 0.24 & 0.000 & 80.256 \\
\hline 0.30 & 0.000 & 45.350 \\
\hline 0.36 & 0.000 & 10.650 \\
\hline 0.42 & 4.329 & 1.830 \\
\hline 0.48 & 18.627 & 0.000 \\
\hline 0.54 & 91.812 & 0.000 \\
\hline
\end{tabular}




\section{CONCLUSION}

The Iris recognition system is developed by using image processing toolbox of Matlab software. In this, we used Hough transform technique to find the outer and inner regions of Iris. After that applied rubber sheet technique to the segmented Iris for converting from circular region into a rectangular region. In feature encoding, the normalized Iris is encoded and forms binary bit of Iris template by using Gabor filter technique. In feature matching, defines the integrity match score by using hamming distance technique to the two encoded Iris templates. In feature matching, the encoded Iris template is compared with database eye Image of Iris template and generated the matching score by using Hamming distance technique. Based on the matching score, we get the result. The future research work is to implement this project for real time personal authentication for security purpose.

\section{REFERENCES}

[1] C. Houston, "Iris Segmentation and Recognition Using Circular Hough Transform and Wavelet Features," pp. 1-4, 2010.

[2] M. S. Monaheng and K. Padmaja, "Iris Recognition using Circular Hough Transform," International Journal of Innovative Research in Science, Engineering and Technology, ISSN: 2319-8753, vol. 2(8), pp. 3546-3553, Aug. 2013.

[3] R.B. Dubey and M. Abhimanyu, "Iris Localization using Daugman's Intero-Differential Operator," International Journal of Computer Applications, ISSN: 0975-8887, vol. 93, no. 3, pp. 6-12, May 2014.

[4] D. Maeva, "Iris Segmentation using Daugman's Integro-Differential Operator," 2010.

[5] W. Yuan and W. He, "A Novel Eyelash Detection Method for Iris Recognition," IEEE Engineering in Medicine and Biology 27th Annual Conference Shanghai, China, pp. 6536- 6539, Sep. 2005.

[6] W. Zhu, Z. Zhao, and Y. Wu, "An Algorithm of Eyelashes Detection for Iris Recognition," International Journal of Security and Its Applications, vol. 10, no. 7, pp. 195-202, 2016.

[7] M.Adam, F.Rossant, F.Amiel, B.Mikovikova, and T. Ea, "Eyelid Localization for Iris Recognition," Radio Engineering, vol. 17, no. 4, pp. 82-85, Dec. 2008.

[8] R. Abduljalil, Z. Nasharuddin, and M. Ismail, "Efficient Iris Segmentation Based on Eyelid Detection," Journal of Engineering Science and Technology, vol. 8, no. 4, pp. 399-405, 2013.

[9] R. Satish, P. Rajesh Kumar, and I. Praneeth, "Iris Recognition System Using Geodesic Active Contours for Non-Ideal Iris Images," International Journal of Engineering Research in Electronics and Communication Engineering, vol. 2(6), pp. 81-87, Jun. 2015.

[10] A. Mohammed, M. Abdullah, S. S. Dlay, and W. L. Woo, "Fast and Accurate Pupil Isolation Based on Morphology and Active Contour," International Journal of Information and Electronics Engineering, vol. 4, no. 6, pp. 418-422, Nov. 2014.

[11] D. S. Jeong, D. Cho, J. Jo, M. K. Bae, M. W. Park, and E. C. Lee, "Compensation for Non-linear Iris Pattern Deformation based on the Tensile Properties of Iris," Wseas Transactions on Information Science and Applications, ISSN: 2224-3402, vol. 12, pp. 315-323, 2015.

[12] Z. Wei, T. Tan, and Z. Sun, "Nonlinear Iris Deformation Correction Based on Gaussian Model," S.-W. Lee and S.Z. Li (Eds.): ICB 2007, pp. 780-789, 2007.

[13] S. Anicham and C. Murukesh, "An Efficient Iris Recognition System Using Contour let Transform and Neural Networks," International Journal of Innovative Research in Science, Engineering and Technology, ISSN: 2319-8753, vol. 3(4), pp. 11876-11881, Apr. 2014.

[14] V. Mayank, S. Richa, and N. Afzel, "Improving Iris Recognition Performance Using Segmentation, Quality Enhancement, Match Score Fusion, and Indexing," IEEE Transactions on Systems, pp. 1$15,2008$.
[15] S. Pardhi and S. G. Qureshi, "Designing and Implementation of Iris recognition System Using Morphological Bridged Canny Edge Detection and KNN Classifier," International Journal of Engineering and Computer Science, ISSN: 2319-7242, vol. 4(6), pp. 12604-12609, Jun. 2015.

[16] P. P. Chitte, J. G. Rana, R. R. Bhambare, V. A. More, R. A. Kadu, and M. R. Bendre, "IRIS Recognition System Using ICA, PCA, Daugman's Rubber Sheet Model Together," International Journal of Computer Technology and Electronics Engineering, ISSN: 22496343, vol. 2(1), pp. 16-23, 2010.

[17] C. Sanchez-Avila and R. Sanchez-Reillo, "Two different approaches for Iris recognition using Gabor filters and multiscale zero-crossing representation," Pattern Recognition, vol. 38, pp. 231-240, 2005.

[18] K. Sathiyaraja, M. Dhineshkumar, and N. Thiyagarajan, "Iris Segmentation and Recognization Using Log Gabor Filter and Curvelet Transform," International Journal of Engineering and Computer Science, ISSN: 2319-7242, vol. 2(9), pp. 2709-2714, Sep. 2013.

[19] E. P. Win and N. Aye, "An Effective Iris Recognition System," International Conference on Advances in Engineering and Technology, pp. 29-30, Mar. 2014.

[20] S. Dalal and T. Sahoo, "A Selective Feature Matching Approach for Iris Recognition," International Journal of Computer Applications, ISSN: 0975-8887, vol. 41, no. 20, pp. 34-39, Mar. 2012.

[21] A. I. Desoky, H. A. Ali, and N. B. Abdel-Hamid, "Enhancing Iris Recognition System Performance using Templates Fusion," Ain Shams Engineering Journal, vol. 3, pp. 133-140, 2012.

[22] K. Sharma and H. Monga, "Efficient Biometric Iris Recognition Using Hough Transform with Secret Key," International Journal of Advanced Research in Computer Science and Software Engineering, vol. 4(7), pp. 632-640, Jul. 2014.

[23] M. W. Amit and R. T. Satish, "Eyelids, Eyelashes Detection Algorithm and Hough Transform Method for Noise Removal in Iris Recognition," International Journal of Computer Applications, ISSN: 0975 - 8887, vol. 112, no. 3, Feb. 2015.

[24] P. Verma, D. Maheedhar, S. Basu, and P. Verma, "Hough Transform Method for Iris Recognition-A Biometric Approach," International Journal of Engineering and Innovative Technology, ISO 9001:2008 Certified, ISSN: 2277-3754, vol. 1(6), PP. 43-48, Jun. 2012.

[25] N. Singh, G. Dilip, and P. S. Krishna, "Iris Recognition System using A Canny Edge Detection and A Circular Hough Transform," International Journal of Advances in Engineering \& Technology, ISSN: 2231-1963, vol. 1(2), pp. 221-228, May 2011.

[26] S. Nithyanandam, K. S. Gayathri, and P. L. K. Priyadarshini, "A New Iris Normalization Process for Recognition System with Cryptographic Techniques," International Journal of Computer Science, ISSN: 1694-0814, vol. 8(4), no. 1, pp. 342-348, Jul. 2011.

[27] C. R. Prashanth, D. R. Shashi kumar, K. B. Raja, K. R. Venu gopal, and L. M. Patnaik, "High Security Human Recognition System using Iris Images," International Journal of Recent Trends in Engineering, vol. 1, no. 1, pp. 647-652, May 2009.

[28] Open source Libraries for eye Images of CASIA database link: http://biometrics.idealtest.org/dbDetailForUser.do?id=4.

[29] Open source code Libraries for Iris Recognition System link1:http://www.peterkovesi.com/studentprojects/libor/sourcecode.h tml.link2:http://www.advancedsourcecode.com/Iris.asp. 